\title{
The cycles of Argentine wine exports / Los ciclos de las exportaciones de vino argentinas
}

\author{
Juan Manuel Cerdá ${ }^{1}$ and y Ricardo Hernández Duarte ${ }^{2}$ \\ ${ }^{1}$ Investigador del CONICET y del Centro de la Argentina Rural, Universidad Nacional de Quilmes, Argentina \\ ${ }^{2}$ Becario CONICET y del Centro de la Argentina Rural, Universidad Nacional de Quilmes, Argentina
}

Resumen. Este trabajo explora los diferentes episodios por los cuales transitó la industria vinícola argentina durante el periodo 1980-2012, en el marco de la globalización del sector. En particular, se estudia la importancia que tuvieron algunos bodegueros en dicho proceso así como el efecto que tuvo el tipo de cambio y los mercados receptores del vino argentino en la expansión de las exportaciones en los últimos años. La hipótesis de este trabajo es que, a partir de los años 80, los bodegueros vieron en las exportaciones una salida a la crisis más importante que atravesó el sector en la Argentina. Esto implicó, necesariamente, una transformación del mismo para producir vinos de calidad que pudieran ser vendidos en el mercado internacional. Así, los bodegueros sumaron a una tradición vitivinícola centenaria nuevas prácticas y estrategias de desarrollo. Por otro lado, a lo largo de 30 años, diversas políticas macroeconómicas influyeron en la expansión del sector en general y en las exportaciones en particular. En síntesis, en este trabajo intentamos demostrar que el crecimiento de las exportaciones de vinos argentinos tuvo un camino sinuoso y diferente a lo que la bibliografía hasta el momento ha encontrado para los países exportadores del "nuevo mundo". El trabajo se basa en diferentes fuentes de información cuantitativas brindadas por el Instituto Nacional de Vitivinicultura (INV), en las estadísticas de la Bolsa de Comercio de Mendoza y la Organización Internacional de la Viña y el Vino (OIV), así como también en entrevistas recogidas en fuentes secundarias a informantes claves del sector.

\begin{abstract}
This paper analyses the different episodes that the Argentina wine industry transited between the years 1980 to 2012, within a context of globalization of the wine-grape industry. In particular, we study the significance that some winemakers had in the process and the effect of the exchange rate and recipients of Argentine wine in the expansion of exports in recent years. The hypothesis of this article is that, from the $80 \mathrm{~s}$, the winemakers observed in exports as a solution to face their most important crisis. This required a transformation of the industry to produce quality wines that could be sold on the international market. Thus, winemakers added to a century-old winemaking tradition new practices and strategies. On the other hand, over 30 years, various macroeconomic policies affect the overall industry growth, especially in exports. On balance, in this paper we propose to show that the growth of exports of Argentine wine was a sinuous and different path to the literature has been found for the "new world" exporters countries. The data was obtained from Instituto Nancional de Vitivinícultura (INV), Bolsa de Comercio de Mendoza and International Organisation of Vine and Wine (OIV) as well as interviews collected on primary and secondary sources.
\end{abstract}

Résumé. Cet article explore les différents épisodes pour lesquels transitaient l'industrie du vin en Argentine pendant la période 1980-2012, dans le contexte de la mondialisation du secteur. En particulier, l'importance qu'ils avaient certains fabricants de vin dans ce processus et l'effet qui avait le taux et les bénéficiaires du marché du vin argentin échange dans l'expansion des exportations au cours des dernières années est étudiée. L'hypothèse de ce travail est que depuis les années 80 , les exportations de vignerons ont vu un moyen de sortir de la plus grande crise que le secteur en Argentine. Cela implique nécessairement une transformation du secteur pour produire des vins de qualité qui pourraient être vendus sur le marché international. Ainsi, les vignerons ont rejoint une tradition viticole nouvelles pratiques centenaires et stratégies de développement. D'autre part, plus de 30 ans, différentes politiques macroéconomiques ont influencé le développement du secteur en général et les exportations en particulier. Bref, dans cet article, nous essayons de montrer que la croissance des exportations de vins argentins avait une façon sinueuse et différente de ce que la littérature jusqu'à présent été trouvée pour les pays exportateurs du 《 nouveau monde ». Le travail est basé sur différentes sources d'informations quantitatives fournies par l'Institut National de Viticulture (INV), les statistiques de la Bourse de Mendoza et l'Organisation Internationale de la Vigne et du Vin (OIV) ainsi que dans les interviews recueillies sur des sources secondaires à des informateurs clés du secteur. 


\section{Introducción}

Desde los inicios de la década de 1990 el sector vitivinícola argentino se ha destacado por un crecimiento sostenido de sus exportaciones. En cierta medida, los estudios han hecho especial énfasis en que ello se debió a la entrada de capitales durante la "convertibilidad"1 que permitió la incorporación de tecnología y la reconversión del sector. Sin embargo, los trabajos científicos no han explorado con suficiente cuidado otros tres factores fundamentales que influyeron sobre las ventas al exterior de vinos. En primer lugar, el cambio engendrado por los productores y las estrategias que éstos diseñaron en las décadas previas al despegue de las exportaciones; en segundo lugar, el papel que jugó el tipo de cambio y, en tercer lugar, el grado de concentración de los mercados y de las empresas exportadoras del sector vitivinícola. El objetivo de este trabajo es demostrar que el crecimiento exportador del sector ha estado asociado a la una conjunción de factores asociados que permiten entender de una manera más compleja su evolución, en un mercado caracterizado por la globalización y la elevada competencia internacional.

La Argentina es un país vitivinícola por historia y por volumen de producción, con importantes niveles de consumo per cápita y que ha comenzado a exportar recién en las últimas décadas. Esta característica lo diferencia de los "nuevos productores" de vino del mundo y explicaría, en parte, por qué el "gigante dormido" (así se la ha denominado por años a la vitivinicultura Argentina) aún tiene un potencial de crecimiento exportador significativo. Las exportaciones de vino se incrementaron entre 1990 y 2015 en $500 \%$ en términos de volumen y en $5.276 \%$ en términos de valor [1]. Asimismo, mientras que las exportaciones representaban el $2 \%$ de la producción total a comienzos de la década de 1990 en los últimos años aquella representa el $20 \%$ del total [2]. A pesar de este desarrollo espectacular la vitivinicultura argentina mantiene al mercado interno como el principal mercado, al menos en términos de volumen.

Si bien gran parte de la bibliografía ha puesto el acento en estas transformaciones, aquí intentaremos mostrar que la reconversión argentina tuvo origen un poco más atrás, por lo menos, a principios de la década de 1980 a partir de una reconversión silenciosa realizada por bodegueros y productores vitivinícolas.

En medio de la crisis más grande que afectó al sector durante toda la década de 1980 algunos bodegueros comenzaron un proceso de modernización que se prolonga hasta nuestros días. Como veremos, éstos fueron favorecidos por políticas económicas que permitieron, en primer lugar, la reconversión del sector en los años noventa y, en segundo lugar, la expansión de las exportaciones de forma significativa a partir de la devaluación de enero del 2002. Pero, nada de todo esto hubiese sido posible sin un cambio en la producción primaria que comenzó en la década de

\footnotetext{
${ }^{1}$ La Ley de Convertibilidad en Argentina (Ley $\mathrm{N}^{\circ}$ 23.928) fue sancionada el 27 de marzo de 1991 por el Congreso de la República Argentina, durante el gobierno de Carlos Menem. Establecía a partir del 1 de abril de 1991 una relación cambiaria fija entre la moneda nacional y la estadounidense que duró hasta el 6 de enero del 2002. El objetivo central de dicha política, donde el tipo de cambio fue usado como ancla nominal de precios, era frenar los altos niveles de inflación que había soportado la economía argentina durante toda la década de 1980.
}

1980, desarrollada por entrepreneur que visualizaron en la exportación una salida a la crisis. Para ello debían transformar su producto, convertirlo en un bien que sea atractivo para los consumidores internacionales y afrontar los riesgos de un mercado cada vez más competitivo. Este último elemento (el riesgo) es importante ya que como veremos las políticas del Estado argentino no tuvieron una clara definición de largo plazo que permitiera a los productores tomar decisiones en base a un horizonte de mediano plazo.

En general, se postula desde la teoría económica que hay una influencia positiva del tipo de cambio sobre las exportaciones de un bien transable como lo es el vino, ésta no parece tener la misma intensidad a lo largo del tiempo. Por el contrario, es posible identificar distintas etapas por las cuales tuvo que atravesar el sector y que, en algunos casos, las exportaciones crecieron a pesar de que el tipo de cambio no los beneficiaba y viceversa. A lo largo de los últimos 40 años, diversas políticas macroeconómicas influyeron en la expansión del sector en general y en las exportaciones en particular. Por lo tanto, parece haber cierto grado de independencia entre el tipo de cambio, los comportamientos de los empresarios y los cambios en la regulación del mismo, aspecto que pretendemos explicar.

En otro sentido, intentaremos mostrar cómo el proceso de concentración de los mercados de destino y de las empresas exportadoras también contribuye a entender las particularidades de la expansión del sector.

El presente trabajo tiene carácter analítico-descriptivo sobre desempeño de las exportaciones de los vinos argentinos, con el fin de captar aquellos factores que estarían explicando dicho proceso con una perspectiva histórica de largo plazo. En esta dirección, se analiza fundamentalmente tres factores: las transformaciones previas al crecimiento de las exportaciones y al origen de las mismas, la influencia del tipo de cambio y el nivel de concentración de los mercados y de las empresas. Asimismo, estos aspectos son indicadores básicos de comercio exterior que proporcionan información sobre una medida de dinamismo comercial [3]. Sin embargo, somos concientes que existen una multiplicidad de otras variables que podrían aportar a la discusión pero que exceden los objetivos de este trabajo, como por ejemplo son: las preferencias del consumidor, acuerdos comerciales, costos de transporte, impuestos al comercio, aumento de costos internos, entre otras.

En particular, se analiza la relación entre las exportaciones con el tipo de cambio como variable proxy de la competitividad de la economía en general y del sector en particular. Es por ello que aquí hemos decidido estudiar la evolución de las exportaciones [1] a la luz del Tipo de Cambio Real (TCR) a partir de la serie realizada por el Banco Central de la República Argentina (BCRA) [4]. También, se analiza la evolución y el cambio de la composición de las exportaciones de vino en términos de valor y volumen a lo largo del período en estudio. Por último, para medir el nivel de concentración de los mercados y de las empresas se utilizó en todos los casos es el Índice de Concentración (IC) [5].

El IC es un indicador básico de comercio exterior que mide el grado de participación de una muestra desagregada en porcentajes. Matemáticamente, es una proporción de un valor entero o decimal entre cero y uno, que puede ser expresado en porcentajes (Durán-Lima, 2008). Para medir el nivel de concentración de destinos de las exportaciones 
de vino está dado por la siguiente fórmula:

$$
I C(n)=\sum_{n} \frac{X_{i}}{X_{t}}
$$

donde:

$n=$ cantidad de destinos

$X_{i}=$ Exportaciones de vino al i-ésimo destino (en hl)

$X_{t}=$ Exportaciones totales $(\mathrm{en} \mathrm{hl})$.

Para el caso de concentración de las empresas exportadoras la fórmula es la siguiente:

$$
I C(n)=\sum_{n} \frac{X_{j}}{X_{t}}
$$

donde:

$n=$ cantidad de empresas

$X_{j}=$ Exportaciones de la j-ésima empresa (en hl)

$X_{t}=$ Exportaciones totales (en hl).

En ambos casos, el IC está dividido en tres medidas el IC1, IC5 e IC10, los cuales miden el grado de concentración acumulado del principal, cinco y diez primeros destinos o empresas, respectivamente.

La construcción de las series de datos se han utilizados diversas fuentes, como son: los informes estadísticos anuales del Instituto Nacional de Vitivinicultura (INV), la información disponible en la página del Observatorio Vitivinícola Argentino (OVA) y del BCRA.

La delimitación temporal (1980-2015) se debe a dos factores fundamentales. Por un lado, en dicho período se presentan los principales cambios en el sector vitivinícola argentino como resultado de la inserción del sector al mercado mundial y las transformaciones internas que debió hacer para ello. Por otro lado, los estudios sobre el sector se han concentrado en el análisis de la década de 1990 identificándosela como el momento de la reconversión de la vitivinicultura argentina. Sin embargo, se ha obviado un análisis de largo plazo que incluya los años de la mayor crisis del sector en toda su historia -la década de 1980- y el desarrollo más reciente.

\section{Los orígenes de la inserción de los vinos argentinos en el mercado mundial}

Como ha sostenido Anderson et. al. (2001) [6] y Anderson (2004) [7], desde finales de los años '60 del siglo pasado se incorporaron al mercado mundial de vinos productores provenientes de países sin tradición vitivinícola: Estados Unidos, Chile, Nueva Zelanda, Australia o Sudáfrica. Estos "nuevos productores" han impulsado el aumento de las exportaciones de vinos dándole forma a un mercado mucho más dinámico y complejo en las últimas décadas. Pero, también, desde comienzos de la década de 1980 , el mercado del vino se vio afectado por una caída del consumo a nivel mundial y, especialmente, de los habitantes de los países consumidores de Europa como son Francia, España o Italia. Estos dos procesos empujaron a los productores tradicionales de vino -llamados también del "viejo mundo"- a buscar nuevos mercados, como por ejemplo: Estados Unidos, Australia, Inglaterra o, más recientemente, China, lo que ha ampliado el intercambio, haciendo así aún más complejo el mercado que en el pasado.

En este marco, algunos trabajos recientes han analizado las diferentes estrategias que desarrollaron los bodegueros del "viejo mundo" con relación a los del "nuevo mundo" o del hemisferio sur ([8-10]). Según estos autores, la característica sobresaliente de los bodegueros de los "nuevos países" -entre los que incluyen a la Argentina-, es que fueron mucho más dinámicos y tuvieron un perfil orientado a la exportación mayor que los europeos. Sin embargo, a nuestro entender, la vitivinicultura argentina es un caso excepcional dentro de los productores calificados como del "nuevo mundo" debido a tres factores fundamentales: en primer lugar, a diferencia del resto de estos países, la producción vitivinícola en la Argentina tiene varios siglos de existencia $[11,12]$. En segundo lugar, como se analizará más adelante, la exportación fue una alternativa a un modelo de desarrollo agotado basado en vinos de baja calidad orientado al mercado interno y no una estrategia productiva explícita orientada al comercio internacional. Por último, más allá del salto cualitativo y cuantitativo que experimentó el vino argentino en el mercado mundial en las últimas décadas, el mercado interno sigue siendo el pilar fundamental para el sector.

En este sentido, si bien el consumo de vino en la Argentina ha caído significativamente en las últimas cuatro décadas - pasando de 92 litros per cápita en 1970 [13] a 24 litros en 2015 [14]- el mercado interno representa aún hoy entre el 75\% / 80\% de la producción total [2]. Esto les permite a los bodegueros manejar alternativas comerciales y estrategias de desarrollo más allá de la producción de vinos para el mercado mundial. A nuestro entender, estos elementos son lo suficientemente importantes como para diferenciar a la vitivinicultura argentina del resto de los productores del "nuevo mundo".

Desde finales del siglo XIX y hasta finales de la década de 1970 la vitivinicultura argentina se había desarrollado exclusivamente basada en el mercado interno. Una producción destinada a un mercado consumidor masivo, con poca competitividad y con alta protección arancelaria, donde el sector realizaba su producto a partir de nociones más prácticas que "científicas", tanto en los aspectos enológicos como agronómicos. La desgravación impositiva de las décadas de 1960 y 1970 junto a un proceso especulativo por parte de algunos grupos económicos importantes potenció la sobreproducción de vinos comunes en el marco de un proceso de caída del consumo a escala nacional. Mientras que el consumo interno se desplomaba la superficie implantada no cesó de crecer a un promedio de 2\% anual entre 1945 hasta 1978. Durante los trece años que duro la crisis (1977-1990) se perdieron aproximadamente 140.000 hectáreas de vides en todo el país, de las cuales 106.000 correspondieron a la provincia de Mendoza. Asimismo, la elaboración de vino disminuyo $9.183 .886 \mathrm{hl}$, de los cuales 7.331.257 hl dejaron de ser producidos en dicha provincia. La crisis representó una caída de $40 \%$ tanto del área cultivada como de la producción de vino en tan solo una década [13]. La transformación del sector comenzó en este contexto, a partir de la acción individual de entrepreneurs que exploraron nuevos mercados como una alternativa a la crisis.

Esto requirió de investigación, transformación empresarial y desarrollos productivos y comerciales que fueron abriendo las puertas de los mercados internacionales 
a los vinos argentinos. En algunos casos fueron emprendimientos nuevos, en otros había experiencia y tradición familiar que sólo necesitaba aggiornarse, mientras que otros aprovecharon la tradición del vino argentino para apostar a su transformación. Pero todos tenían algo en común: importantes volúmenes de capital económico, social y cultural para invertir en un momento dominado por la incertidumbre. Así, la decisión de algunos bodegueros fue crucial. El rediseño organizacional de las firmas, la adopción de tecnología moderna tanto en las bodegas como en el sector primario-, la importación de clones de diversos varietales, la ampliación de la frontera vitícola así como la incorporación del marketing para promocionar vinos en el exterior fueron partes constitutivas de este proceso de transformación vitivinícola. Esto fue acompañado por una política del INV que acompaño dicho proceso. ${ }^{2}$ Las investigaciones sobre mercado internacionales buscando los países más adecuados para los vinos argentinos tienen antecedentes desde finales de los años 70 .

Los volúmenes de exportación pasaron de 71.348 $h l$ en el año 1980 a $445.536 h l$ en el año 1990 [1], lo que representaba tan sólo el $0,7 \%$ y $3,17 \%$ de la producción total $[1,2]$, respectivamente. Si bien estos valores eran insignificantes, muestran que algunos productores comenzaron a ver a las exportaciones como una válvula de escape a la crisis. Se debe recordar que los vinos argentinos tenían una mala reputación y ello impedía la entrada a los mercados internacionales. Por lo tanto, no sólo se necesitó de un cambio en la calidad sino también de la reversión de dicha imagen.

Estos cambios se dieron en el marco de la crisis y como parte de un proceso de modernización. Esta nueva vitivinicultura se orientó en búsqueda de la calidad, basada en el perfil de los vinos varietales del nuevo mundo. Los conocimientos desarrollados en otras latitudes fueron adaptados a las necesidades y características locales y, en gran medida, esto fue hecho a partir de la sistematización y estudios realizados por los propios productores. Así, la utilización de clones, la maceración prefermentativa en frío, la fermentación maloláctica, la utilización de barricas -que reemplazaron o complementaron la utilización de las piletas de hormigón y toneles tradicionales-, el deshoje, el cambio en la conducción de las vides, el estudio sistemático del clima, el reconocimiento del terroir, el estrés hídrico y el marketing, pasaron a formar parte del discurso de la transformación vitivinícola, originando un cambio copernicano para el sector.

En efecto, durante la década 1980 se implantaron 19.000 hectáreas nuevas en todo el país, lo que representaba el 9\% de las vides existentes en 1990, de las cuales más del $60 \%$ correspondieron a la provincia de Mendoza [15]. Esto indica que, más allá de la crisis, se dio una reconversión productiva importante en el sector vitícola que era fundamental para producir el cambio hacia la calidad [16].

Los bodegueros que optaron por la exportación debieron enfrentarse con la imagen negativa que tenía el vino argentino en el exterior y un consumidor mucho más exigente. La característica predominante de los vinos argentinos -alta graduación alcohólica y baja calidad

\footnotetext{
${ }^{2}$ El INV es un instituto público creado en 1959 con el fin de controlar y regular el sector.
}

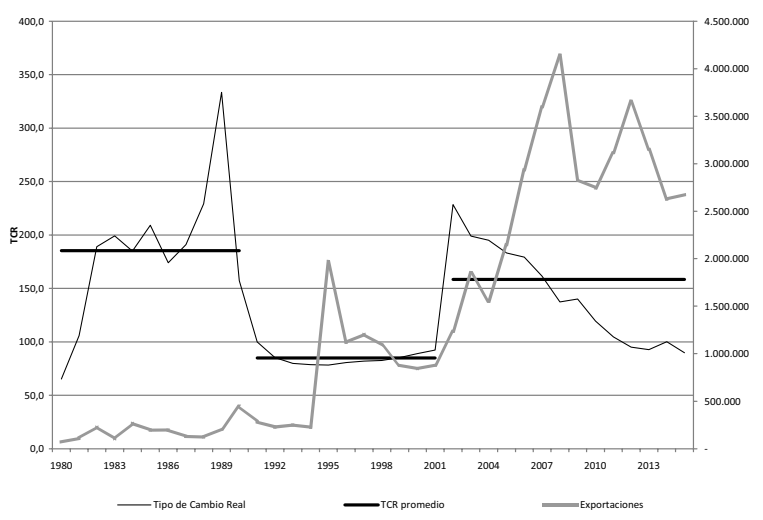

Gráfico 1. Evolución de las exportaciones de vinos argentinos (en $h l$ ) y del Tipo de Cambio Real $(1991=100)$.

enológica-, hacía que su inserción en el mercado mundial sea muy difícil. El mercado norteamericano fue el elegido por los primeros exportadores, en general, no sólo porque se presentaba como el mercado más dinámico sino también porque se acercaba a las características del vino argentino: vinos jóvenes, más aromáticos y con poco paso por barrica.

A continuación, se analiza la evolución de las exportaciones, teniendo en cuenta el tipo de cambio real y el grado de concentración de las mismas.

\section{Fortalecimiento y concentración de las exportaciones del vino argentino}

\subsection{Factores asociados a la dinámica exportadora}

El proceso de expansión de las exportaciones vitivinícolas de los años 90 ha sido explicado, en general, por la modernización del sector y por el aumento de la competitividad asociado a un Tipo de Cambio Real (TCR) elevado. Por otro lado, también, la pérdida de competitividad, producto de una apreciación del TCR en los últimos años, ha sido el argumento más utilizado para explicar la desaceleración de las exportaciones. Sin embargo, deberíamos decir que estos son sólo dos aspectos que explican en parte dicho proceso.

Asimismo, debe considerarse que el ciclo productivo de la vitivinicultura dura un año y que las decisiones en términos de inversión no pueden ser medidas en función del corto plazo. A diferencia de otros productos primarios el costo de implantación de la vid es relativamente alto y el retorno de la inversión necesita de al menos cinco años. ${ }^{3}$ Por otro lado, como ya fue señalado, el crecimiento de las exportaciones de vino comenzó tímidamente a finales de los años '80, registra un salto importante a mediados de los años '90, y se hizo mucho más dinámico a partir de la devaluación del año 2002 (gráfico 1).

En general, desde la teoría económica, se plantea que existe una relación positiva entre un tipo de cambio real elevado y las exportaciones netas [17]. Sin embargo, como se observa en el gráfico 1 , a pesar de haber tenido un tipo de cambio elevado durante toda la década de 1980 las exportaciones de vino en esos años fueron

\footnotetext{
${ }^{3}$ Esto se debe a que la vid comienza a producir recién a los tres o cuatro años de ser implantada y que sus resultados dependen en gran medida de las condiciones climáticas.
} 


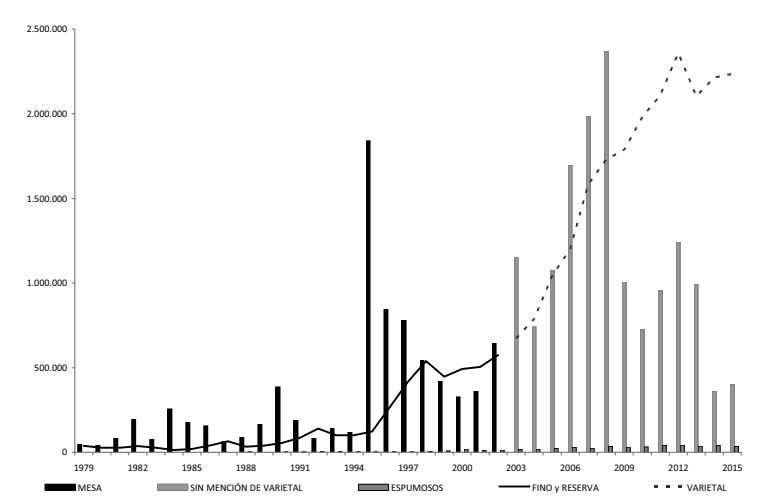

Gráfico 2. Evolución de las exportaciones según clase de vinos, en $h l$.

insignificantes. Esto se debió a la mala imagen (y calidad) que hacía poco probable su colocación en el mercado internacional. Esto comenzó a revertirse hacia final de la década y comienzos de la siguiente, aún antes de la implementación del tipo de cambio fijo en 1991. De hecho, durante los años 80, los volúmenes exportados se multiplicaron por seis y se triplicaron en términos de valores. Si bien parece poco con relación a lo que sucedió con posterioridad, podemos afirmar que marca el comienzo del proceso de exportación. Fue, entonces, durante estos años y el primer quinquenio de los noventa donde los nuevos vinos argentinos se insertaron en el mercado mundial y comenzaron, lentamente, a revertir su imagen negativa.

A partir de marzo de 1991, la apreciación de la moneda junto a la eliminación de los aranceles para la importación de bienes de capital y la caída del precio internacional del acero permitieron que gran parte del sector comenzara a importar bienes de capital destinados a modernizar las bodegas y los viñedos. Como lo han señalado Azpiazu y Basualdo (2000) [18], estas medidas favorecieron la importación de tanques de fermentación, levaduras, barricas y clones, y aceleró la entrada de capitales nacional y extranjero al sector vitivinícola. A su vez, una coyuntura particular -la sequía que afectó a la vitivinicultura española de 1995 produjo una demanda extraordinaria de los vinos argentinos que lo posicionó en un nivel superior a los registros históricos- explica el salto cuantitativo de las exportaciones en 1995. Más allá de esta coyuntura, las exportaciones se sostuvieron en torno al millón de hectolitros a lo largo de toda la segunda mitad de los años noventa. Este aumento benefició particularmente a los vinos de mesa (vinos "comunes") aunque, también, impulsó a los vinos finos y reserva, como analizaremos con mayor detalle en el próximo apartado (gráfico 2).

A lo largo de los casi once años que duró la convertibilidad (desde marzo de 1991 a diciembre de 2001) con un TCR muy bajo las exportaciones casi se triplicaron en términos de volumen y se multiplicaron casi por ocho en términos de valor. A partir de este momento los nuevos vinos argentinos empiezan a ser conocidos en el mundo, ya no sólo por los especialistas -que los comenzaron a premiar-, sino también por el consumidor casual que busca probar nuevos varietales. Sin embargo, la expansión más significativa de las exportaciones todavía estaba por producirse.

Como era de esperar, la devaluación de enero de 2002 tuvo efectos positivos sobre la vitivinicultura, al igual que resto de los bienes transables. A pesar de algunos primeros análisis pesimistas [19], el sector reaccionó de forma positiva y el mercado mundial mostró un creciente interés por el vino argentino. En menos de dos años, los volúmenes exportados se volvieron a duplicar y llegaron a su nivel máximo en 2008 (gráfico 1). O sea, en el período comprendido entre 2002 y 2008 , las exportaciones crecieron de forma significativa, aumentado a una tasa anual de $19 \%$ en términos de volumen y de $25 \%$ en términos de valor. Sin embargo, el año 2008 parece haber marcado un techo en las exportaciones de vino argentino. A partir de 2009 los volúmenes exportables disminuyen y su tendencia aparece menos clara. Durante el período 2008-2015 las exportaciones cayeron 35\% en volumen, pero crecieron $32 \%$ en términos de valor (gráfico 1 y cuadro 1).

La crisis internacional del 2008 y la caída de las ventas a Rusia parecerían ser las razones más importantes detrás de la caída de los volúmenes exportados. En este sentido, este país había sido el mayor destino de las ventas de vino desde el 2005 y cede su lugar a Estados Unidos quien llega a concentrar más del $40 \%$ de las exportaciones en el 2011, como veremos en el próximo apartado. Un hecho interesante a destacar es que, en medio de la crisis internacional, la caída en términos del volumen fue superada por un aumento en términos de valor. Esto indica que, en promedio, las exportaciones aumentaron crecieron en calidad durante este período aún con un tipo de cambio que siguió apreciándose, apenas por encima de la década de 1990.

En síntesis, en una perspectiva de largo plazo las exportaciones crecieron desde finales de la década de 1980; muy lentamente al comienzo pero, luego, con mayor intensidad a mediados de los años 90 y de forma espectacular desde la devaluación del 2002. Por lo tanto, las exportaciones han estado aumentando desde hace más de 30 años, tanto bajo en períodos de tipo de cambio apreciado -como en la convertibilidad o durante los últimos años-, o con un tipo de cambio alto como fue durante gran parte de la primera década del siglo XXI.

Con esto, no se pretende negar la incidencia que puede tener esta variable en algunos momentos específicos sino que se intenta matizar el poder explicativo de ésta sobre las ventas al exterior. Para entender mejor la evolución de las exportaciones analizaremos a continuación el perfil de vino y la composición de los mercados externos durante estos últimos treinta años, lo que influyó, en alguna medida, sobre la evolución de las exportaciones.

\subsection{Cambios en la composición de las exportaciones y los nuevos mercados}

Brevet et al. (2014) [20] señalan que en el año 2011 el $86 \%$ del volumen y el $76 \%$ del valor de las exportaciones de vino argentino fraccionado en botella correspondían al segmento Premium (USD FOB 14 - USD FOB 60) y que sólo el $2 \%$ del valor de las exportaciones se constituía de segmentos de valores inferiores. De la misma manera, estos autores indican la creciente presencia y notoriedad que ha alcanzado el malbec argentino en el mundo, posicionándose como el varietal insignia de la industria nacional. En efecto, la comercialización de vinos de calidad pasó a asumir un papel protagónico en las exportaciones de los últimos años, tanto en precio como en 
Cuadro 1. Evolución de las exportaciones de vino.

\begin{tabular}{|l|l|l|l|l|l|}
\hline & 1990-2015 & 1990-2001 & 2001-2015 & 2001-2008 & 2008-2015 \\
\hline Volumen & $500,38 \%$ & $97,88 \%$ & $203,41 \%$ & $369,65 \%$ & $-35,40 \%$ \\
\hline Valor & $5276,50 \%$ & $876,55 \%$ & $450,56 \%$ & $318,10 \%$ & $31,68 \%$ \\
\hline
\end{tabular}

volumen. Debido al cambio en la clasificación de los vinos establecida por el Instituto Nacional de Vitivinicultura en el 2003 no es posible hacer un análisis comparativo de todo el período pero, a pesar de esto, es posible observar cómo la composición de las exportaciones ha ido cambiando en el tiempo. ${ }^{4}$

En el gráfico 2 se aprecia cómo los vinos “de mesa”, en el contexto de bajos volúmenes de exportaciones totales, constituyeron dos tercios de los volúmenes exportados durante la década de 1980 y comienzos de 1990. Por su parte, los vinos denominados "finos y reserva" comenzaron lentamente a crecer desde inicios de los años 1990 pero recién pudieron igualar en volumen a los "vinos de mesa" en la segunda mitad de la década. En este sentido, sólo en el período 1999-2001 los vinos "finos y reservas" superaron en volumen a los vinos de mesa. Para el año 2000 la Argentina exportó $843.023 h l$ de los cuales el $58,4 \%$ eran de "finos y reserva" y $38,8 \%$ de vino de mesa, lo que estaría indicando un cambio en el perfil de los vinos argentinos exportados.

Si observamos la composición de las exportaciones en volumen a partir de la nueva denominación implementada en el año 2003, los vinos varietales representaron el 36,4\% mientras que los no varietales $62,3 \%$ para dicho año, imponiéndose una tendencia durante toda la década hacia la preferencia de estos últimos. Estos llegaron a representar más del 80\% de las exportaciones en los años 2014-2015, mientras que los vinos no varietales explican apenas entre el 10-15\% de las mismas (gráfico 2).

Sin embargo, si se analizan las exportaciones en términos de valor (gráfico 3), se puede observar que si bien los vinos "de mesa" y los "no varietales" crecieron, lo hicieron a un nivel muy inferior al de los "vinos finos" y "varietales". Mientras que los vinos de mesa representaban el 45\% del valor de las exportaciones en 1990, para el 2002 sólo lo hacían en $13 \%$. Por su parte, los vinos no varietales que representaban el $25,5 \%$ del valor de las exportaciones en el 2003 redujeron su participación a tan sólo el 7\% en el 2015. Por el contrario, las exportaciones de vino varietales han crecido de forma sostenida desde el 2003 hasta el 2015, sin registrar efecto alguno de la crisis internacional. Esto se explica por un aumento del valor promedio de estos vinos, lo que lleva a pensar que el aumento del precio

\footnotetext{
${ }^{4}$ La resolución $\mathrm{N}^{\circ} \mathrm{C} 12 / 2003$ del INV determinó que a partir de la liberación al consumo de los vinos de la cosecha 2004 los vocablos "de mesa" y "fino" quedan sin validez para utilizarse como indicativos de calidad diferencial de los mismos, de esta manera, la denominación legal que rige en la identificación del producto es el término "vino" seguido de la característica cromática. Debido a lo anterior, la comparación de la producción y exportación según tipo de vinos en todo el periodo 1990-2012 se ve limitada. A pesar de la tendencia que se observa entre los vinos denominados "finos y reserva" con vinos identificados como "varietales" no es posible establecer que se esté hablando del mismo tipo de producto. Algo similar sucede entre los vinos denominados "de mesa" y los "no varietales".
}

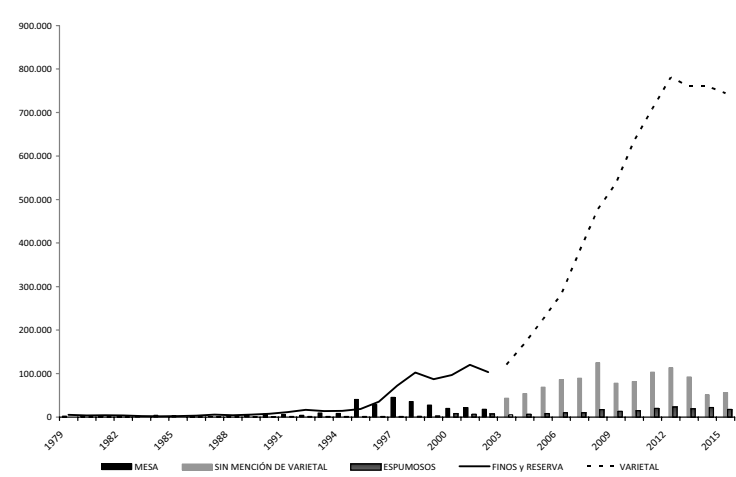

Gráfico 3. Valor de las exportaciones de vinos en miles de dólares corrientes.

unitario de los vinos exportados ha compensado la caída en los volúmenes.

Por último, si nos concentramos en la evolución de los últimos años, podremos observar que lo vinos no varietales tuvieron una retracción más notoria que los varietales a partir 2008, tanto en volumen como en valor. Esto permite afirmar que hay un cambio cualitativo en la composición de los vinos que la argentina exporta al exterior. Esto, en parte, parece estar asociado a los mercados de destino que, como veremos a continuación, se han concentrado en países donde la preferencia por el consumo de vinos varietales ha sido mayor en los últimos años, dejando atrás otros mercados como Rusia o China donde predominaban las exportaciones de vinos sin mención varietal.

En síntesis, como fue mencionado en el apartado anterior, el tipo de cambio ha tenido una importancia relativa $-y$ no lineal- en la evolución de las exportaciones. En este sentido, parecería que el tipo de cambio tuvo una mayor incidencia sobre los no varietales que en los varietales, ya que éstos han podido mantener su posicionamiento en el mercado internacional. Sin embargo, hay otros factores que también explican el desarrollo del sector como, por ejemplo, los cambios impulsados por los productores, transformaciones en el conocimiento y su implementación práctica así como también cambios en los hábitos y gustos de los consumidores, aspecto que excede ampliamente este trabajo. A continuación veremos cómo fue la evolución de las exportaciones según el grado de su concentración (1).

Esta concentración en los mercados de destino está fortalecida por la entrada al mercado estadounidense. Mientras que en 1996 los vinos argentinos que llegaban al país del norte representaba el $4 \%$ del volumen de las exportaciones totales -ocupando la séptima posición como país destino de los vinos argentinos-, a partir del año 2004 se convirtió en el principal importador, representando el $15 \%$ del volumen total de las exportaciones, alcanzando su máxima participación en el 2012 con el $47 \%$ del total y en los últimos años está en torno del $40 \%$. El resto de países que componen los cinco principales destinos (IC5) varían su participación año a año sin un patrón claro. En la última 


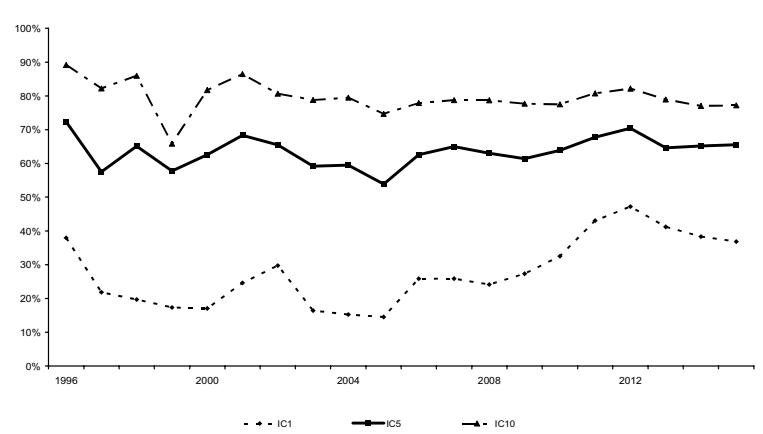

Gráfico 4. IC de exportaciones de vino.

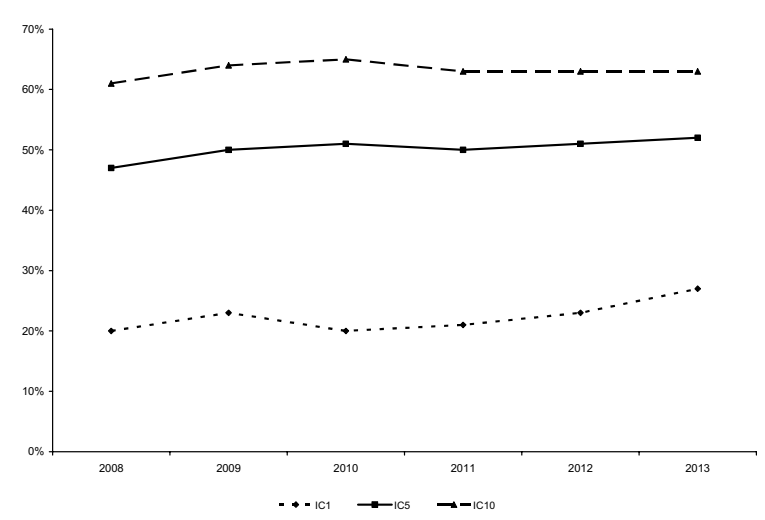

Gráfico 5. Evolución del IC de las empresas exportadoras de vino argentinos.

década, se han consolidado como destino Canadá, Rusia, Reino Unido y Paraguay, que para el 2012 representaban el $8 \%, 6 \%, 5 \%$ y $4 \%$, respectivamente, del total del volumen de las exportaciones. Cabe agregar que, para el mismo año, el $82 \%$ de las exportaciones tenían como destino 10 países (IC10). O sea, se agregan a los anteriores: Brasil, Países Bajos, Japón, Dinamarca y Alemania, que con la participación de cada uno del 4\%, 3\%, 2\%, 1\%, $1 \%$ del total de las exportaciones de vino (INV, 1997, 2000, 2005, 2012). Esta evolución de las exportaciones hacia los mercados más competitivos confirma la existencia de un mayor acercamiento al gusto de los consumidores y permite suponer que ha sido la demanda de estos países los que ha sostenido las exportaciones de vinos varietales en contraposición a los no varietales en los últimos años.

Por otro lado, un proceso similar al de concentración de los mercados se observa a nivel de las empresas y/o grupos exportadores (2), tal como se muestra en el gráfico 5. La principal empresa y/o grupo exportador (IC1) pasó de concentrar el $20 \%$ del volumen de las exportaciones de vino en el año 2008 al $27 \%$ en el año 2013. Por su parte, 10 empresas (IC10) explican más del $60 \%$ de exportaciones totales de vino de la Argentina. En una agroindustria que contaba en dicho año con 950 bodegas podemos afirmar que las exportaciones estaban fuertemente concentradas en un número pequeño de grupos y/o empresas.

Cuando se analiza el IC empresaria sólo para el caso del principal destino -Estados Unidos-, se observa que si bien disminuyó la concentración en el IC1, aumentó de forma proporcional la participación de IC5 (gráfico 6). Esto estaría indicando una mayor participación de las cinco empresas exportadoras de vinos a los Estados Unidos,

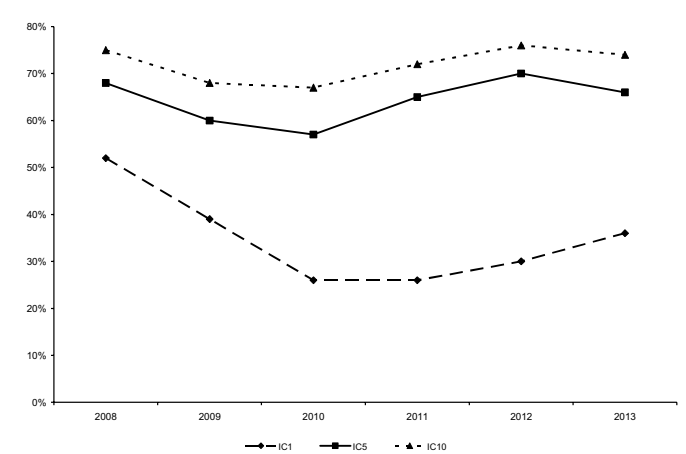

Gráfico 6. Evolución de la IC de las empresas exportadoras de vinos argentinos en los Estados Unidos.

aún cuando la primera sigue dominando el $30 \%$ de las exportaciones totales a dicho país. Esta preferencia (o concentración) de los exportadores por colocar sus vinos en el mercado estadounidense implica cierta dependencia que, en un contexto de crisis como fue la del 2008-2009, pudo haber tenido incidencia sobre el comercio exterior.

En síntesis, esto también estaría reforzando la idea que la apuesta al mercado norteamericano que comenzaron algunos exportadores a finales de los años 80 se ha intensificado en los últimos años, como fue el caso de la bodega Catena Zapata entre otras.

\section{Conclusiones}

El objetivo de este trabajo ha sido analizar el proceso de crecimiento de las exportaciones que se dio en forma de episodios en los últimos 35 años en la Argentina, intentando captar aquellos factores que estarían explicando dicho proceso. Se ha llegado a la conclusión que el aumento de las exportaciones fue el resultado de un proceso de causalidad múltiple y compleja. El primer "episodio" se dio con la reconversión productiva impulsada por algunos empresarios innovadores en el marco de la mayor crisis del sector en la década de 1980 en la Argentina y de la gran expansión de las exportaciones a nivel mundial.

El modelo desarrollado que sostuvo al sector vitivinícola argentino desde finales del siglo XIX hasta mediados de la década de 1970 se había basado en la producción de vinos comunes destinados a un mercado interno que estaba altamente protegido. La crisis de finales de los años '70 no sólo provocó la eliminación de 140.000 ha. de vides en todo el país sino que también fue el comienzo de un fuerte proceso de reconversión productiva. Algunos bodegueros optaron por la exportación como una salida a la crisis pero necesitaban transformarse y adaptarse a la demanda del mercado internacional para poder lograrlo. El bajo precio de los viñedos argentinos en términos internacionales en la década de 1980 y 1990 se planteó como una clara oportunidad para invertir, considerando que en el país se contaba con viñedos de alta calidad enológica, con capital cultura y social importante y con una infraestructura que, aunque desactualizada, permitiría proyectar cambios y sumarse al proceso de globalización de la vitivinicultura.

La estrategia de algunos productores fue sumar a su experiencia y tradición vitivinícola centenarias técnicas de producción y marketing adquiridas en el extranjero -en particular en los Estados Unidos-, y adaptarlas a 
las características y posibilidades locales. Para estos bodegueros las exportaciones de vino operaron como el horizonte que guio la reconversión del sector pero sin que ello se convierta en el principal destino de su producción. En cierta medida, esto fue producto de la mala imagen que tenía el vino argentino y la poca experiencia que tenían los bodegueros para exportar su producto, lo que no les permitió aprovechar plenamente la ventaja competitiva que el bajo valor real de la moneda vigente durante esta década generaba para los bienes transables. En síntesis, en la década de 1980, parecería que la decisión microeconómica de salir al mercado internacional fue una válvula de escape de la crisis, con el fin de revertir el ciclo más negativo que tuvo el sector en todo el siglo XX.

El segundo episodio, se corresponde con el período de la convertibilidad (1991-2001) donde se produjo una fuerte entrada de capitales al sector y un incremento de las exportaciones. Como se ha demostrado, una paradoja de este período es que aún con un TCR bajo las exportaciones crecieron $\mathrm{y}$, en promedio, fueron más elevadas que las de la década anterior. Esto da por tierra la idea de que las exportaciones dependen exclusivamente del tipo de cambio y permite observar que las transformaciones previas comenzaron a dar sus resultados cambiando la imagen del vino argentino $\mathrm{y}$, por lo tanto, generando una mayor demanda del mercado mundial.

Luego de la devaluación del 2002, la historia parece repetirse. Durante los primeros años luego de la salida de la convertibilidad el TCR fue alto (2002-2008) y las exportaciones de vino aumentaron de forma significativa. Luego de ello 2008-2015) el TCR comenzó a retrasarse y las exportaciones comenzaron un sendero de menos claro. Por un lado cayeron los volúmenes pero por otro lado se incrementaron las exportaciones en valor. Por tanto, no parece sería ser el tipo de cambio el único causante de dicho freno.

A nuestro entender, la crisis internacional del 2008 hizo que las exportaciones tuvieran un pequeño retroceso. Como hemos visto esto podría estar asociado a una fuerte concentración -por mercados y por empresas- de las exportaciones de vinos argentinos hacia el mercado estadounidense y la pérdida del mercado ruso. Los vinos varietales muestran una menor elasticidad frente a estos hechos lo que estaría indicando que los exportadores siguen apostando a mercados externos a pesar de la caída de la competitividad. Nuevamente, como durante la convertibilidad, parecería que los exportadores una vez que ganan mercados resisten retirarse de ellos debidos a los costos de entrada y al efecto reputación.

En síntesis, la dinámica exportadora de largo plazo sugiere que el tipo de cambio no alcanza para explicar por si sólo todo el proceso y que otros factores como la imagen, el tipo de producción o las estrategias microeconómicas impactan sobre las posibilidades de desarrollo del sector. La capacidad de imponer un producto específico como el vino en un mercado globalizado y tan competitivo implica un esfuerzo importante por parte de las empresas. De todas maneras, con niveles más estables y previsibles del tipo de cambio real debería esperarse que las condiciones mejoren para un sector que, por las características del cultivo y de la producción, requiere un horizonte más estable en el mediano y largo plazo.

Por último, no debemos olvidar que la vitivinicultura argentina sigue basada en un mercado interno que representa hoy el $70 \%$ del total de la producción. Esto le permite al sector no depender exclusivamente de las exportaciones y tener una red de contención que es el mercado interno -que sigue estando fuertemente protegido, con niveles de consumo per cápita elevados en términos internacionales.

\section{Referencias}

[1] INV, Anuarios de exportaciones años 1990-2015, http://www.inv.gov.ar/index.php/men-esta disticas/men-estadisticas-vitivinicolas/ 16-cat-estadisticas/63-est-menu-anual-ex porta, acceso en: feb. 2016

[2] INV, Síntesis básica de estadísticas vitivinícolas argentinas. Años 1993-2015, http://www.inv. gov.ar/inv_contenidos/pdf/estadisticas/te speciales/2016/SINTESIS_B\%C3\%80SICA_1993_ __2015.pdf, acceso en: feb. 2016

[3] J. Durán-Lima, Indicadores de comercio exterior y política comercial: generalidades metodológicas $e$ indicadores básicos (CEPAL, Santiago de Chile, 2008)

[4] BCRA, Estadísticas: ITCRM, http://www.bcra. gov.ar/PublicacionesEstadisticas/Indice_ tipo_cambio_real_multilateral.asp, acceso en: abril 2016

[5] OVA. Dinamismo comercial: una medida de diversificación/concentración de mercados y actores, http://www . observatoriova.com/informes/d inamismo.html, acceso en: feb. 2016

[6] K. Anderson, D. Norman, and G. Wittwer, CIES, 0143 (2001)

[7] K. Anderson, The World's Wine Markets. Globalization at work (Edward Elgar, Cheltenham, 2004)

[8] K. Anderson y S. Nelgen, Global wine markets, 1961 to 2009: a statistical compendium (Adelaide, 2011)

[9] J. Martínez Carrión y F. Medina Albaladejo, SEHA, 12-03 (2012)

[10] F. Medina Albaladejo, J. Martínez Carrión y J. Ramon-Muñoz, Am. Lat. Hist. Econ., 21, 2 (2014)

[11] P. Lacoste, El vino del inmigrante. Los inmigrantes europeos y la industria vitivinícola argentina: su incidencia en la incorporación, difusión y estan darización del uso de topónimos europeos 1852-1980 (CEM, Mendoza, 2003)

[12] P. Lacoste, Mendoza, Cultura y economía, (Caviar Bleu, Mendoza, pág. 57-114, 2004)

[13] OVA, Datos y estadísticas de superficie, cosecha y elaboración de vinos de Argentina, con aperturas para Mendoza y San Juan. Evolución desde 1960 hasta 2001, http: //observatoriova . bolsamza . com.ar/BOE/OpenDocument/1203201241/OpenD ocument/opendoc/openDocument.jsp, acceso en: feb. 2016

[14] Instituto Nacional de Vitivinicultura, Salidas de vino autorizadas para consumo interno, http://www . inv.gov.ar/inv_contenidos/pdf/estadistic as/anuarios/2015/Cuadros_salidas_definiti vas_2015.pdf, Acceso en: feb. 2016

[15] INV, V Censo Vitícola Nacional, Mendoza (1990)

[16] J. M. Cerdá y R. J. Hernández-Duarte, Sujetos sociales del agro argentino. Configuraciones históricas 
y procesos de cambio (Prohistoria, Rosario, pág. 249271, 2013)

[17] R. Frenkel, Rev. Econ. Pol. BA, 3 y 4, (2008)

[18] D. Aspiazu y E. Basualdo, El complejo vitivinícola argentino en los noventa: Potencialidades y restricciones (CEPAL, Buenos Aires, 2000)
[19] D. Aspiazu y E. Basualdo, Terceras Jornadas de Estudios Agrarios y Agroindustriales, (Buenos Aires, 2003)

[20] X. Brevet; J. E. Orrego and A. Gennari, AAWE, 166 (2014) 